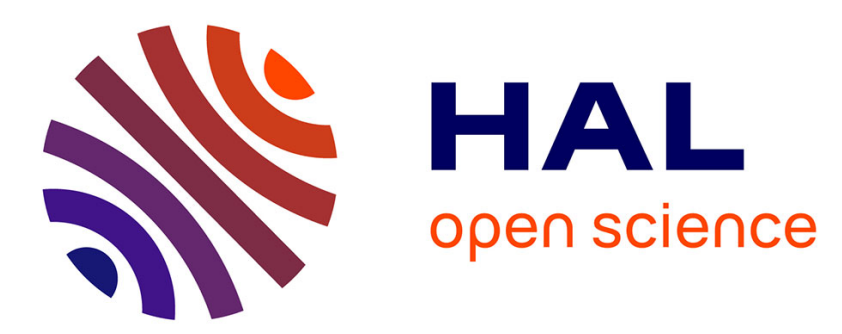

\title{
Local Structure Study About Co in a-Axis Films of YBa2(Cu0.93Co0.07)3O7- $\delta$ Using Polarized XAFS
}

F. Bridges, E. Bauer, C. Booth, Z. Kvitky, Z. Trajanovic, I. Takeuchi, C. Lobb, T. Venkatesan

\section{- To cite this version:}

F. Bridges, E. Bauer, C. Booth, Z. Kvitky, Z. Trajanovic, et al.. Local Structure Study About Co in aAxis Films of YBa2(Cu0.93Co0.07)3O7- $\delta$ Using Polarized XAFS. Journal de Physique IV Proceedings, 1997, 7 (C2), pp.C2-1093-C2-1095. 10.1051/jp4:19972145 . jpa-00255207

\section{HAL Id: jpa-00255207 https://hal.science/jpa-00255207}

Submitted on 1 Jan 1997

HAL is a multi-disciplinary open access archive for the deposit and dissemination of scientific research documents, whether they are published or not. The documents may come from teaching and research institutions in France or abroad, or from public or private research centers.
L'archive ouverte pluridisciplinaire HAL, est destinée au dépôt et à la diffusion de documents scientifiques de niveau recherche, publiés ou non, émanant des établissements d'enseignement et de recherche français ou étrangers, des laboratoires publics ou privés. 


\title{
Local Structure Study About $\mathrm{Co}$ in $a$-Axis Films of $\mathrm{YBa}_{2}\left(\mathrm{Cu}_{0.93} \mathrm{Co}_{0.07}\right)_{3} \mathrm{O}_{7-\delta}$ Using Polarized XAFS
}

\author{
F. Bridges, E.D. Bauer, C.H. Booth, Z. Kvitky, Z. Trajanovic*, I. Takeuchi*, C.J. Lobb* \\ and T. Venkatesan* \\ Physics Department, University of California, Santa Cruz, CA 95064, U.S.A. \\ * The Center for Superconductivity Research, Department of Physics, University of Maryland, \\ College Park, MD 20742, U.S.A.
}

\begin{abstract}
We present polarized XAFS data for quenched and slow cooled $a$-axis thin films of YBCO. The oxygen peak is the same for both the $a$ and $b$ axes and does not change with cool-down rate. This suggests that Co acts as an oxygen getter in the YBCO film.
\end{abstract}

\section{INTRODUCTION}

Shortly after the superconductor $\mathrm{YBa}_{2} \mathrm{Cu}_{3} \mathrm{O}_{7-5}(\mathrm{YBCO})$ was discovered, $\mathrm{Co}$, Fe and other similar dopants were added to $\mathrm{YBCO}$ in order to probe the differences between the plane and chain $\mathrm{Cu}$ sites and as a general probe of superconductivity. Early experiments established that $\mathrm{Co}$ and $\mathrm{Fe}$ substitute for $\mathrm{Cu}$, primarily on the chain $[\mathrm{Cu}(1)]$ site, and this result is now well accepted. A number of XAFS experiments indicated that, although the nearest neighbor oxygen atoms are well ordered, the further neighbor environment, composed of $\mathrm{Ba}$ and $\mathrm{Cu}$ atoms, is distorted or disordered $[1,2,3,4]$. These experiments and other experiments have been interpreted in terms of a $<110>$ off-center displacement of some or all of the $\mathrm{Co}$ (or Fe) [1,5]. Mössbauer experiments indicate at least three distinct sites for $\mathrm{Fe}$ in terms of the quadrupolar splittings [6]. One site with a small fractional occupancy $(<20 \%)$ has a small quadrupolar splitting indicating a nearly symmetric site. The other two sites have larger splittings and comparable occupancy. Similar sites are expected for Co.

In spite of the extensive investigations of $\mathrm{YBCO}: \mathrm{Co}$ and $\mathrm{YBCO}: \mathrm{Fe}$, the $\mathrm{Co}$ and $\mathrm{Fe}$ sites are still not well determined. It is clear from the Mössbauer studies that there is more than one local environment about Fe. It is also clear that changing the sample preparation (which likely changes the $\mathrm{O}$ concentration) can change $\mathrm{T}_{c}$ significantly [7].

Recently, high quality $a$-axis films of YBCO:Co have been prepared using $\mathrm{LaSrGaO}_{4}$ substrates [8]. This offers a new opportunity to investigate the local structure about $\mathrm{Co}$, by using $\mathrm{x}$-rays polarized along the $a$-, $b$-, and $c$-axes. In particular, we can check whether the distances are the same along each axis and the amount of $O$ on each axis. Here we present polarized XAFS results for two $a$-axis thin film samples of $\mathrm{YBCO}: \mathrm{Co}$, deposited on $\mathrm{LaSrGaO}_{4}$, and compare with previous $c$-axis films deposited on $\mathrm{LaAlO}_{3}$. One sample was quenched while the other was cooled slowly to room temperature to vary $\mathrm{T}_{c}$ at the same dopant concentration (7\%). X-ray diffraction indicates less than 5\% a-axis orientation for the $c$-axis film and less than $10 \%$ twinning of the $b$ and $c$ axes in the $a$-axis film.

\section{EXPERIMENTAL DETALS}

Polarized XAFS data were collected on beamline 4-3 at SSRL using $\mathrm{Si}<220>$ monochromator crystals. The thin films were aligned either perpendicular to the $x$-ray beam or at near grazing incidence (within $9^{\circ}$ ), depending on the sample orientation and desired polarization direction. For the $a$-axis films, $\boldsymbol{P}$ is in the film plane for $\boldsymbol{P}$ along either the $b$-or $c$-axis and perpendicular to the film when it is parallel to $a$. For the $c$-axis films, $P$ is in the film plane for $a b$-plane polarization and perpendicular to the film when parallel to $c$. Note that a misorientation of $9-10^{\circ}$ introduces only $3 \%$ of the orthogonal component, which is negligible for these measurements.

\section{RESULTS}

\subsection{Nearest Neighbors}

In Fig. 1 and 2, we show the Co $K$-edge r-space data for the two $a$-axis films and the $c$-axis film. Several qualitative results are immediately obtained from an inspection of these figures. First, the nearest neighbor oxygen-peak for $\boldsymbol{P}$ in the $a b$ plane is well defined and essentially the same for both the $a$ - and $c$-axis films. In addition, for the $a$-axis films, the first neighbor peak amplitude is the same for both $a$ and $b$ x-ray polarizations and does not change significantly between fast and slow cooled 
treatments. From our earlier analysis of $c$-axis films [9], the amplitude of this peak corresponds to two $O$ atoms in the $a b$-plane $\left(S_{0}{ }^{2}=0.75\right)$; the present results are in agreement, with one near $O$ neighbor on each of the $a$-and $b$-axes. If there are somewhat more $\mathrm{O}$ neighbors about $\mathrm{Co}$ in the $a b$ plane than for the normal $\mathrm{Cu}(1)$ site, then either the amplitude of the first $\mathrm{O}$ peak should be higher or another $\mathrm{CO}-\mathrm{O}$ peak at a longer distance should exist. In our fits, a peak corresponding to about $0.5-0.7 \mathrm{O}$ atoms is found at a longer bond distance; the amplitude of this peak also changes significantly with the fast and slow cooled sample treatments.

Second, the Co-O bond is slightly shorter along the c-axis than in the $a b$-plane by about $0.05 \AA$; note the position of the peak in the real part of the FT in Fig. 1 and 2. A similar but slightly larger difference was obtained for the $c$-axis films. This indicates that the $\mathrm{Co}$ atom is in a region that is epitaxial with the $\mathrm{YBCO}$. The fact that the shorter bond is along the $c$-axis both when it is in the film plane ( $a$-axis films) and when it is perpendicular to the substrate ( $c$-axis films), indicates that the Co is incorporated into the YBCO and not into some oxide precipitate that is epitaxial with the substrate. For a Cu(1) substitution site, these results are consistent with the average tetragonal structure for XBCO:Co films as well as with the general features of models of the local Co environment that have been developed from earlier XAFS studies of powder samples [1,4]. Note that these results are inconsistent with a primary $\mathrm{Cu}(2)$ substitution site which has two $\mathrm{O}$ neighbors each, along the $a$ - and $b$-axes; however, we cannot rule out a small fractional occupancy of the $\mathrm{Cu}(2)$ site at the 10-20\% level.

For $\boldsymbol{P}$ parallel to the $c$-axis, the amplitude of the nearest neighbor $O$-peak is larger than for $\boldsymbol{P}$ along the $a$ or $b$-axcs. However, there is a considerable discrepancy in the amplitude of this peak between the $a$-axis and $c$-axis films. Along the $c$-axis, two $O$ neighbors are expected for the $\mathrm{Cu}(1)$ site; thus the amplitude should be twice that for the $a$ - or $b$-axis polarizations. The amplitude of the Co-O peak for the $c$-axis film (and most of the other $c$-axis films studied earlier) is consistent with two neighbors, while the amplitude of this peak for both of the a-axis films corresponds to approximately $1.5 \mathrm{O}$ neighbors. This likely indicates more disorder of the axial oxygens but might possibly be the result of a larger concentration of $\mathrm{Co}$ on the $\mathrm{Cu}(2)$ site in $a$-axis films.

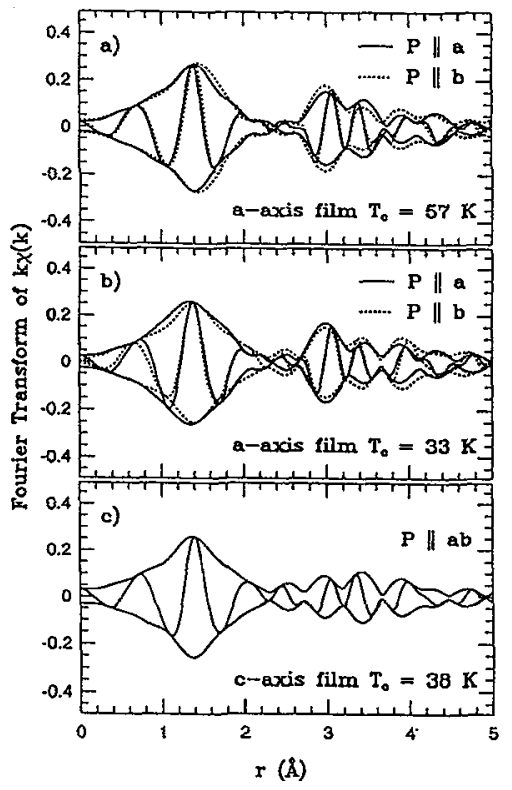

FIG. 1. A comparison of the Co $K$-edge data for the $a$ and $c$-axis films with $P$ in the $a b$ plane; a)the quenched a-axis film, b) the slow cooled a-axis film, and c) the $c$-axis film. FT range is 3.5-11.5 $\AA^{-1}$, Gaussian broadened by $0.3 \AA^{-1}$. The fast oscillatory curve is the real part of the $F T_{\text {, while the }}$ amplitude is $\left((\mathrm{Re})^{2}+(\mathrm{Im})^{2}\right)^{1 / 2}$.

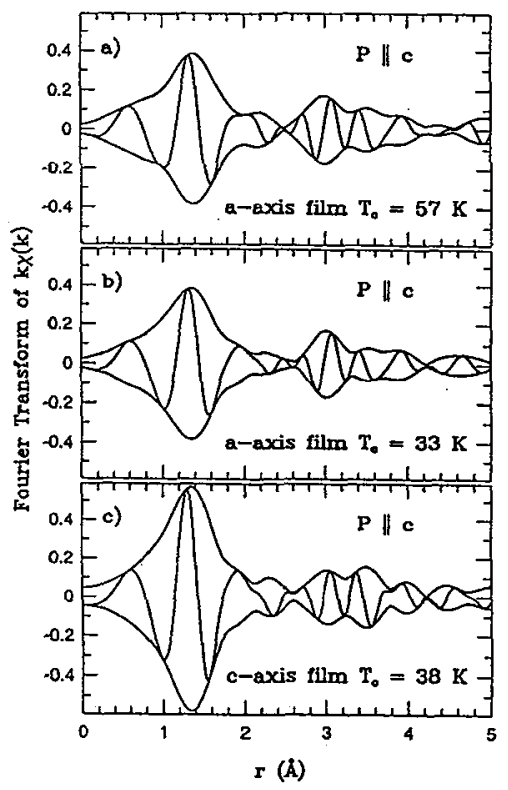

FIG. 2. A comparison of the Co $K$-edge data for the $a$ - and $c$-axis films with $P$ along the $c$-axis a) the quenched a-axis film, b) the slow cooled a-axis film, and c) the $c$-axis film. Same FT range as for Fig. 1. 


\subsection{Further neighbors}

The further neighbor multi-peak, formed of $\mathrm{Co}-\mathrm{Ba}$ and $\mathrm{Co}-\mathrm{Cu} / \mathrm{Co}$ peaks, is much smaller than expected from $\mathrm{Cu} \mathrm{K}$-edge studies of pure $\mathrm{YBCO}$ as found previously in power samples $[1,4]$. Consequently, the $\mathrm{Co}$ is in a $\mathrm{Cu}(1)$ site that is strongly disordered or distorted relative to the $\mathrm{Ba}$ and $\mathrm{Cu} / \mathrm{Co}$ neighbors. The amplitude of this multi-peak is larger for the $a$-axis films than for the $c$-axis films. The further neighbor environment could not be fit well for the latter because of the low amplitude. Fits of the Co$\mathrm{Ba}$ and $\mathrm{Co}-\mathrm{Cu} / \mathrm{Co}$ were carried out assuming $50 \%$ of the $\mathrm{Co}$ are displaced along the $\langle 110\rangle$ direction [1]. We included constraints in the fit such that the off-center displacement of the Co was consistent with both the contraction of the Co-O bond and the long and short $\mathrm{Co}-\mathrm{Ba}$ pair distances. We were able to obtain reasonable fits to the data for the quenched sample, but the overall Co-Ba amplitude is low, (about $5 \mathrm{Ba}$ neighbors for $\mathrm{S}_{0}{ }^{2}=0.7$ ) if not constrained. Fair fits for all the $a$ and $b$ polarization data sets can be obtained with a fixed overall $\mathrm{Co}-\mathrm{Ba}$ amplitude corresponding to 8 neighbors. In these fits the average $\mathrm{Co}-\mathrm{Ba}$ distance is contracted and the off-center displacement of the $C_{0}$ is approximately $0.2 \AA$. This is suggestive of a high $O$ concentration since for pure YBCO the Co-Ba distance is short for full oxygenation and long for oxygen depletion. Further analysis using other models is now in progress.

\section{DISCUSSION AND CONCLUSIONS}

The two different treatments of the $a$-axis films result in a large variation of $T_{c}$ yet the Co XAFS are only slightly changed, primarily in the further neighbor region. It is expected that the $O$ concentration and distribution within the chains will change between the fast and slow cooled sample preparations; the lack of change in the XAFS for the first neighbor peak indicates, however, that the number of $\mathrm{O}$ neighbors about $\mathrm{Co}$ is unchanged within experimental error. Consequently $\mathrm{Co}$ appears to act as an oxygen getter, keeping the number of nearest $O$ neighbors constant. If the average $O$ concentration changes then there must be large changes in the rest of the plane. Note that if $7 \%$ Co is incorporated into YBCO on the chain sites, $21 \%$ of the $\mathrm{Cu}(1)$ sites will be occupied by $\mathrm{Co}$. The gettering action of $\mathrm{Co}$ may result in an $\mathrm{O}$-depleted region surrounding small Co clusters. A moderate average $\mathrm{O}$ depletion of 0.2 (i.e. $\mathrm{O}_{6.8}$ ) would result in a large $\mathrm{O}$-depletion $\left(\mathrm{O}_{6.6}\right)$ around the remaining $\mathrm{Cu}$ in the chains (here we assume $2.5 \mathrm{O}$ about each $\mathrm{Co}_{\mathrm{O}}$ on average). This may be part of the reason for the large change in $\mathrm{T}_{c}$ with sample preparation at a fixed dopant level. If so, the fast cooled sample must have a higher $O$ concentration than the slow cooled sample which seems counter-intuitive. However, recent results on YBCO:Fe powders shows that $T_{c}$ is highest in the highly oxygenated samples, and has the shortest $\mathrm{Co}-\mathrm{Ba}$ pair distances [10].

For the Co-Ba peak, the data are consistent with a significant fraction of the Co off-center along the $<110>$ directions. At $7 \%$ $\mathrm{Co}, \mathrm{Co}$ pairs and some triplets are expected assuming only a random distribution. If there is some tendency for clustering some longer Co chains should form; we have found that a zig-zag Co-chain model along the $<110>$ direction (as used earlier) fits the data reasonably well but further analysis using other models is in progress.

\section{Acknowledgments}

The experiments were performed at the Stanford Synchrotron Radiation Laboratory, which is operated by the U.S. Department of Energy, Division of Chemical Sciences, and by the NIH, Biomedical Resource Technology Program, Division of Research Resources. This work is supported in part by NSF grant DMR-92-05204.

[1] F. Bridges et al., Phys. Rev. B 39, 11603 (1989).

[2] C. Y. Yang et al., Phys. Rev. B 42, 2231 (1990).

[3] B. D. Padalia et al., J. Phys. Condens. Matter 4, 6865 (1992).

[4] H. Renevier et al., Phys. Rev. B 47; 1398 (1993).

[5] H. Renevier et al., Physica C 230, 31 (1994).

[6] C. Blue et al., Phys. Rev. B 37, 5905 (1988).

[7] F. Bridges, J. B. Boyce, and R. I. Johnson, App. Phys. Lett. 60, 3042 (1992).

[8] Z. Trajanovic et al., IEEE Trans. Appl. Superconductivity 5, 1237 (1995).

[9] E. D. Bauer, F. Bridges et al. (unpublished).

[10] F. Bridges, E. Bauer et al. (unpublished). 